\title{
GLOBAL WELLPOSEDNESS TO THE INCOMPRESSIBLE MHD EQUATIONS WITH SOME LARGE INITIAL DATA
}

\author{
XIAOPING ZHAI
}

Abstract. In this paper, we mainly study the global wellposedness for the $n$-dimensional homogeneous and nonhomogeneous incompressible magnetohydrodynamic equations in the critical Besov spaces. By fully using the advantage of weighted function generated by heat kernel and Fourier localization technique, we first get the global wellposedness for the homogeneous incompressible MHD equations with initial data under a nonlinear smallness hypothesis. It is amazing that we can exhibit an initial data satisfying that nonlinear smallness assumption, despite each component of the initial data could be arbitrarily large. Then, as an application of our global well-posedness, we also extend our result to the inhomogeneous incompressible MHD equations.

Mathematics subject classification (2010): 35Q35, 76D03, 76W05.

Keywords and phrases: Global wellposedness, MHD equations, Besov space.

\section{REFERENCES}

[1] H. ABIDI AND M. PAICU, Existence globale pour un fluide inhomogéne, Ann. Inst. Fourier (Grenoble)., 57, 3 (2007), 883-917.

[2] H. ABIDI AND M. PAICU, Global existence for the magnetohydrodynamic system in critical space, Proc. Roy. Soc. Edinburgh Sect. A., 138, 3 (2008), 447-476.

[3] H. Bahouri, J. Y. Chemin and R. Danchin, Fourier Analysis and Nonlinear Partial Differential Equations, Grundlehren Math. Wiss. 343, Springer-Verlag, Berlin, Heidelberg, 2011.

[4] J. Bourgain And N. Pavlović, Ill-posedness of the Navier-Stokes equations in a critical space in 3D, J. Funct. Anal., 255, 9 (2008), 2233-2247.

[5] C. CAO AND J. WU, Global regularity for the 2D MHD equations with mixed partial dissipation and magnetic diffusion, Adv. Math., 226, 2 (2011), 1803-1822.

[6] J.Y. Chemin And I. Gallagher, On the global wellposedness of the 3-D Navier-Stokes equations with large initial data, Ann. Sci. École Norm. Sup., 39, 4 (2006), 679-698.

[7] J.Y. Chemin AND I. GallaGHeR, Wellposedness and stability results for the Navier-Stokes equations in $\mathbb{R}^{3}$, Ann. Inst. H. Poincaré Anal. Non Linéaire., 26, 2 (2009), 599-624.

[8] J.Y. Chemin, D.S. McCormickm, J.C. Robinson And J.L. Rodrigo, Local existence for the non-resistive MHD equations in Besov spaces, arXiv:1503.01651v1 [math.AP].

[9] Q. CHEN, Z. TAN AND Y.J. WANG, Strong solutions to the incompressible magnetohydrodynamic equations, Math. Methods Appl. Sci., 34, 1 (2011), 94-107.

[10] Q. CHEN, C. MiAO AND Z. ZHANG, On the regularity criterion of weak solution for the $3 D$ viscous magnetohydrodynamics equations, Comm. Math. Phys., 284, 3 (2008), 919-930.

[11] R. DANCHIN, Local theory in critical spaces for compressible viscous and heat-conducting gases, Comm. Partial Differential Equations, 26, 7-8 (2007), 1183-1233.

[12] P.A. Davidson, An Introduction to Magnetohydrodynamics, Cambridge University Press, Cambridge, 2001.

[13] B. DESJARDINS AND C. LE BRIS, Remarks on a nonhomogeneous model of magnetohydrodynamics, Differential Integral Equations, 11, 3 (1988), 377-394. 
[14] G. Duvaut And J. L. Lions, Inéquations en thermoélasticité et magnétohydrodynamique, Arch. Ration. Mech. Anal., 46, 4 (1972), 241-279.

[15] C.L. FefFerman, D.S. MCCORMICKM, J.C. Robinson AND J.L. Rodrigo, Higher order commutator estimates and local existence for the non-resistive MHD equations and related models, $\mathrm{J}$. Funct. Anal., 267, 4 (2014), 1035-1056.

[16] H. Fujita And T. Kato, On the Navier-Stokes initial value problem I, Arch. Ration. Mech. Anal., 16, 4 (1964), 269-315.

[17] F. CHARVE AND B. HASPOT, Existence of strong solutions in a larger space for the shallow-water system, Adv. Differential Equations., 17, 11-112 (2012), 1085-1114.

[18] J.F. GERBEAU AND C. LE BRIS, Existence of solution for a density-dependent magnetohydrodynamic equation, Adv. Differential Equations, 2, 3 (1997), 427-452.

[19] P. Germain, The second iterate for the Navier-Stokes equation, J. Funct. Anal., 255, 9 (2008), 2248 2264.

[20] G. GuI, Global well-posedness of the two-dimensional incompressible magnetohydrodynamics system withvariable density and electrical conductivity, J. Funct. Anal., 267, 5 (2014), 1488-1539.

[21] C. He AND X. XIN, Partial regularity of suitable weak solutions to the incompressible magnetohydrodynamic equations, J. Funct. Anal., 277, 1 (2005), 113-152.

[22] C. HE, X. HuAng AND Y. WANG, On some new global existence results for the 3D magnetohydrodynamic equations, Nonlinearity., 27, 2 (2014), 343-352.

[23] J. HuAng, M. Paicu AND P. Zhang, Global solutions to 2-D inhomogeneous Navier-Stokes system with general velocity, J. Math. Pures Appl., 100, 6 (2013), 806-831.

[24] X. HUANG AND Y. WANG, Global strong solution to the 2-D nonhomogeneous incompressible MHD system, J. Differential Equations, 254, 2 (2013), 511-527.

[25] D. IfTIMIE, The resolution of the Navier-Stokes equations in anisotropic spaces, Rev. Mat. Iberoamericana, 15, 1 (1999), 1-36.

[26] T. KATO, Strong $L^{p}$ solutions of the Navier-Stokes equations in $\mathbb{R}^{n}$ with applications to weak solutions, Mathematische Zeithschrift, 187, 4 (1984), 471-480.

[27] H. Koch ANd D. TAtaru, Well-posedness for the Navier-Stokes equations, Adv. Math., 157, 1 (2001), 22-35.

[28] H. Kozono And M. Yamazaki, Semilinear heat equations and the Navier-Stokes equation with distributions in new function spaces as initial data, Comm. Partial Differential Equations, 18, 5-6 (1994), 959-1014.

[29] F. Lin And P. Zhang, Global small solutions to MHD type system (I): 3-D case, Comm. Pure Appl. Math., 67, 4 (2014), 531-580.

[30] F. LIN, L. XU AND P. ZHANG, Global small solutions to 2-D incompressible MHD system, J. Differential Equations., 259, 10 (2015), 5440-5485.

[31] Q. LIU AND S. CUI, Well-posedness for the incompressible magneto-hydrodynamic system on modulation spaces, J. Math. Anal. Appl., 389, 2 (2012), 741-753.

[32] C. MiAO AND B. YUAN, $n$ the well-posedness of the cauchy problem for an MHD system in Besov spaces, Math. Meth. Appl. Sci., 32, 1 (2009), 53-76.

[33] M. PAICU AND P. ZhANG, Global solutions to the 3-D incompressible inhomogeneous Navier-Stokes system, J. Funct. Anal., 262, 8 (2012), 3556-3584.

[34] J. Peetre, New Thoughts on Besov Spaces, Duke Univers. Math. Ser., vol. 1, Duke University, Durham, NC, 1976.

[35] X. Ren, J. WU, Z. XiAng AND Z. Zhang, Global existence and decay of smooth solution for the 2-D MHD equations without magentic diffusion, J. Funct. Anal., 267, 2 (2014), 503-541.

[36] W. Burnside, Modélisation mathématique et simulation numérique, Paris Milan Barcelone: Masson, 1994.

[37] M. Sermange And R. Temam, Some mathematical questions related to the MHD equations, Comm. Pure Appl. Math., 36, 5 (1983), 635-664.

[38] R. WAN, On the uniqueness for the 2D MHD equations without magnetic diffusion, Nonlinear Analysis: R.W.A., 30, 8 (2016), 32-40.

[39] R. WAN, Global well-posedness to the 3D incompressible MHD equations with a new class of large initial data, arXiv:1509.07660v1.

[40] T. YonedA, Ill-posedness of the 3D-Navier-Stokes equations in a generalized Besov space near $B M O^{-1}$, J. Funct. Anal., 258, 10 (2010), 3376-3387. 
[41] X. ZHAI, Y. LI AND W. YAN, Global well-posedness for the 3-D incompressible inhomogeneous MHD system in the critical Besov spaces, J. Math. Anal. Appl., 432, 1 (2015), 179-195.

[42] X. ZHAI, Y. Li AND W. YAN, Global well-posedness for the 3-D incompressible MHD equations in the critical Besov spaces, Commun. Pure Appl. Anal., 14, 5 (2015), 1865-1884.

[43] T. ZHANG, An elementary proof of the global existence and uniqueness theorem to 2-D incompressible non-resistive MHD system, arXiv:1404.5681v1 [math.AP].

[44] Y. ZHOU, Remarks on regularities for the 3D MHD equations, Discrete Contin. Dyn. Syst., 12, 5 (2005), 881-886. 